\title{
Saber qué y saber cómo. Una propuesta explicativa de tres técnicas terapéuticas empíricamente validadas
}

\section{Knowing What and How. An Explicative Proposal for Three Empirically Validated Therapeutical Techniques}

\author{
Raymundo González-Terrazas ${ }^{1}$ y María Xesús Froxán Parga ${ }^{2}$ \\ ${ }^{1}$ ITECOC, Instituto de Terapias Cognitivo-Conductuales, México \\ ${ }^{2}$ Universidad Autónoma de Madrid, España
}

\begin{abstract}
Resumen: La eficacia demostrada de algunos procedimientos terapéuticos no siempre va seguida de una explicación coherente de su funcionalidad. En este artículo nos proponemos revisar tres de las estrategias terapéuticas más utilizadas en la actualidad: reestructuración cognitiva, defusión cognitiva y mindfulness, ofreciendo una explicación alternativa a las terapias tradicionales, partiendo de su definición como mayoritariamente verbales. La consideración de la cognición como una conducta cualitativamente diferente a cualquier otra conducta humana implica caer en una forma de pensar dualista que poco tiene que ofrecer al desarrollo científico de la psicología. Nuestra propuesta adopta un enfoque monista, basado en procesos de aprendizaje respondiente y operante, dentro de una tradición de análisis conductual. Se considera que las técnicas citadas operan sobre el comportamiento privado pero no difieren ontológicamente del público y, por tanto, están sujetas a contingencias en el proceso terapéutico de forma similar a cualquier otro comportamiento.
\end{abstract}

Palabras clave: reestructuración cognitiva, defusión cognitiva, mindfulness, perspectiva analítico-conductual.

\footnotetext{
Raymundo González-Terrazas (iD) https://orcid.org/0000-0003-4052-7109 es psicólogo en ITECOC, Instituto de Terapias Cognitivo Conductuales Clínica y Docencia de México.

María Xesús Froxán Parga (iD https://orcid.org/0000-0002-6175-4637 es psicóloga y docente en la Facultad de Psicología de la Unviversidad Autónoma de Madrid.

Para citar este artículo: González-Terrazas, R. y Froxán, M. X. (2021). Saber qué y saber cómo. Una propuesta explicativa de tres técnicas terapéuticas empíricamente validadas. Clínica Contemporánea, 12(3), Artículo e25. https://doi.org/10.5093/cc2021a22

La correspondencia sobre este artículo debe enviarse a Raymundo González-Terrazas, ITECOC-Instituto de Terapias Cognitivo Conductuales, C. Horacio Nelson \#15B, Fracc. Costa Azul, 39850, Acapulco, Guerrero, México. E-mail: psic.raygonzalez@gmail.com 


\begin{abstract}
The proven efficacy of certain therapeutical procedures is not always followed by a consistent explanation of their functionality. In this article, we set out to review three of the most used therapeutical strategies today: cognitive restructuring, cognitive defusion and mindfulness, offering an alternative explanation to traditional therapies, starting from their definition as being mostly verbal. The consideration of cognition as a behavior qualitatively different from any other human behavior implies falling into a dualistic way of thinking that offers little to the scientific development of psychology. Our proposal adopts a monist approach, based on respondent and operant learning processes, within a behavioral analysis tradition. It is considered that the cited techniques operate over private behavior but do not differ ontologically from public behavior and, therefore, are subject to contingencies in the therapeutical process similarly to any other behavior.

Keywords: cognitive restructuring, cognitive defusion, mindfulness, behavioral analysis perspective
\end{abstract}

A lo largo de los últimos veinte años, los estudios comparativos de la Asociación Americana de Psicología (APA, 2006) respecto a los resultados de las distintas terapias psicológicas se han decantado, una y otra vez, hacia la mayor validación empírica de las intervenciones cognitivo-conductuales. Las terapias cognitivo-conductuales son hoy por hoy las terapias que cuentan con mayor investigación y aplicaciones para múltiples problemas psicológicos (Echeburúa et al, 2010), tanto que se consideran un sinónimo de las psicoterapias basadas en la evidencia (Hernández-Pineda et al., 2020), pero en distintas ocasiones se han puesto en tela de juicio varios de sus procedimientos debido a una latente insatisfacción para algunos autores referida a la explicación proporcionada cuando se indaga en su naturaleza y en por qué funciona lo que funciona. El modelo cognitivo-conductual ha dado lugar a una enorme cantidad de procedimientos terapéuticos los cuales tienen, al menos en lo que a sus orígenes se refiere, una base común: estar asentados en los principios psicológicos de aprendizaje operante y respondiente. Pero a lo largo del tiempo, las terapias cognitivo-conductuales han ido experimentando una gran cantidad de cambios e innovaciones que condujeron al alejamiento de sus principios fundacionales y a la proliferación de procedimientos y estrategias que intentaban dar una rápida solución a los múltiples problemas con los que se encontraba el clínico en su quehacer profesional (Arbinaga-Ibarzábal, 2000; Mañas, 2007; Tortosa y Mayor, 1997). Con el tiempo, el porqué y el cómo funcionaban estas técnicas se consideró irrelevante mientras que se consiguiese un cambio clínico en el comportamiento objetivo de las personas. Y tampoco hay que olvidar que una gran parte de las técnicas cognitivas fue desarrollada por autores ajenos al modelo conductual y carentes de su rigurosidad experimental (Fatih y Hakan, 2012; Landriscini, 2006; Oller, 2009).

La ya famosa tercera generación de terapias cognitivo-conductuales supuso un freno a este desarrollo desmedido, abogando por un regreso a los orígenes conductuales de los procedimientos técnicos, junto con una depuración teórica y un esfuerzo por la clarificación conceptual (Hayes, 2004; Pérez-Álvarez, 2006). Las preguntas en torno a si el reconocimiento de la eficacia y eficiencia de los procedimientos conductuales sería suficiente para el desarrollo de una tecnología científica, si es importante saber por qué funciona lo que funciona o si es necesario una teoría sólida que enmarque los problemas que se planteen en el desarrollo tecnológico son algunas de las propuestas actuales que consideramos que hay que responder.

En este trabajo nos proponemos revisar tres de los procedimientos terapéuticos más utilizados en la actualidad: el primero de ellos es la reestructuración cognitiva, un clásico dentro del modelo cognitivo-conductual y que ha demostrado sobradamente su gran potencia terapéutica; en segundo lugar, la defusión cognitiva, uno de los elementos centrales de la ACT (Barraca, 2011; Hayes et al., 2006; Luciano et al., 2005), terapia de las denominadas de tercera generación; y, por último, también utilizada y, en ocasiones, ubicada dentro de esta tercera generación, el mindfulness, una estrategia con varias finalidades, entre ellas la misma defusión y que además es enormemente utilizada no solo en el campo clínico sino en otros mu- 
chas áreas de la psicología aplicada (empresas, deporte, salud, etc.) (Larrubia, 2019; Moñivas et al., 2012; Solé et al., 2014; Palmi y Solé, 2016). El objetivo de la revisión que proponemos es ofrecer una visión crítica de las explicaciones que hay detrás de la eficacia de tales estrategias, cuya utilidad queda fuera de toda duda, analizándolas como procedimientos eminentemente verbales y sujetos a las mismas leyes y principios que cualquier otra conducta (encubierta o manifiesta); entendemos que este proceder no invalida la eficacia ni lo conocido acerca de las técnicas citadas, menos aún el modelo terapéutico en el cual se desarrolla, sino que añadiría un mayor rigor experimental, facilitaría el estudio de dichas técnicas así como la manipulación de las variables responsables de su eficacia para orientarlas hacia mejoras que maximicen el éxito terapéutico.

Las tres técnicas que vamos a analizar trabajan con las llamadas cogniciones, abordadas como conducta verbal encubierta (pensar), si bien cada una de ellas lo hace de manera muy diferente, tanto en cuanto a sus objetivos como a los procedimientos para modificarlas.

Por lo que respecta a la reestructuración cognitiva, otorga un estatus causal a los pensamientos, partiendo de una postura filosófica conocida como mentalismo (Moore, 1995; Zilio y Carrara, 2008) que asume la existencia de estados mentales dentro del organismo que difieren a la naturaleza de los comportamientos observables. El mentalismo es una postura filosófica teleológica de causalidad que pone sobre la mesa dos problemas fundamentales: uno concerniente a la ontología de lo mental (qué es lo mental) y en qué se diferencia de lo físico; y un segundo problema relativo a su estatus explicativo (qué papel juega lo mental en la explicación de la conducta) (Skinner, 1985). Pero la consideración de ambos problemas supone caer en una trampa conceptual, ya que se concibe lo mental en los mismos términos que lo físico, es decir, serían dos mundos que coexisten (Ryle, 2005). Frente a esta postura dualista, el conductismo radical es monista y considera los eventos privados como objeto de estudio, con el mismo estatus ontológico y epistemológico que la conducta manifiesta. El pensamiento (es decir, habla interna) se operativiza como conducta verbal encubierta y es una parte más del fenómeno a explicar y nunca su causa (Freixa, 2003). Esto es, no se niega la existencia de los eventos privados, sino que se propone estudiar esos eventos bajo los mismos principios, herramientas y procedimientos como se haría con cualquier otra conducta pública y no como algo que opera distinto a los demás comportamientos.

La adopción de una postura mentalista genera graves problemas a la hora de explicar satisfactoriamente por qué funcionan las terapias cognitivas, ya que los supuestos cambios cognitivos que ocurren y explican, a juicio de sus autores, los cambios en el comportamiento manifiesto solo pueden ser inferidos a través de dicho comportamiento o de la expresión verbal (Froján-Parga y Calero-Elvira, 2011; Froján et al., 2017).

Respecto a la defusión cognitiva, como procedimiento terapéutico incluido en la ACT, se explica desde la Teoría de los Marcos Relacionales la cual, a juicio de su principal autor, sería perfectamente compatible con los planteamientos filosóficos del conductismo radical (Hayes, 2004); sin embargo, esta teoría es muy controvertida, considerada por algunos autores ininteligible en algunos de sus planteamientos y problemática en cuanto a sus aspectos conceptuales, lógicos y teóricos, hasta el punto de que los mismos consideran que está muy alejada del conductismo radical ya que su planteamiento podría ser dualista y sus conceptos mediacionales (Burgos, 2004; Dymond et al., 2010; Gross y Fox, 2009; Tonneau, 2001).

Por último, el mindfulness proviene de una tradición muy diferente y antigua, el budismo zen (Hayes, 2006; Moñivas et al., 2012; Pérez-Álvarez, 2001), y concretamente en una forma de meditación llamada Vipassana, orientada a ver las cosas como son y no como parecen ser. Este procedimiento se ha convertido en los últimos tiempos en algo esencial de los tratamientos psicológicos, argumentando su novedad y beneficios dentro de los modelos terapéuticos que encajan en la llamada tercera generación (Davis y Hayes, 2011; Hayes, 2006); sin embargo, las terapias de tercera generación no son las primeras ni las únicas en incluir en sus procedimientos los acercamientos budistas (Hayes, 2002), sino que existen otras aproximaciones no-conductuales que también lo integran y otras más que tratan de explicar su equivalencia en términos operantes-funcionales (De Silva, 1984).

Una vez presentadas las tres técnicas, vamos a presentar las explicaciones que tradicionalmente se han ofrecido sobre cada una de ellas para a continuación proponer una alternativa explicativa de su funcionamiento, fundamentada en los principios de aprendizaje. 


\section{Explicaciones tradicionales de las técnicas analizadas}

\section{Reestructuración Cognitiva}

Las Terapias Cognitivo-Conductuales (TCC) o terapias conductuales de segunda generación son el conjunto de terapias que más aplicaciones clínicas tienen hoy en día, sobre las que se han desarrollado múltiples protocolos de intervención para diversos tipos de trastornos o problemas psicológicos y que cuentan con los criterios establecidos para considerarse una psicoterapia con una evidencia de moderada a fuerte (Llobell et al, 2004; Stevens y Méndez, 2007; Vera-Villarroel, 2004). No hay duda, como decíamos antes, de que el conjunto de técnicas o procedimientos de la TCC son bastante efectivas y ampliamente utilizadas y, muy probablemente, es por esas mismas razones que la reflexión teórica y el conocimiento fundacional analítico-experimental del quehacer terapéutico se fueron olvidando, respaldados por una mera efectividad empíricamente demostrada (Froján-Parga y Calero-Elvira, 2011).

La reestructuración cognitiva ( $\mathrm{RC}$ ) es un procedimiento muy utilizado por los terapeutas adscritos al modelo TCC y que ha demostrado su efectividad en múltiples estudios sobre una gran variedad de problemas psicológicos (Butler et al, 2006; Caro, 2013; Valadez, 2002). Sin embargo, no existe una definición ni un procedimiento consensuado a la hora de aplicarla. Por el contrario, existen tantas propuestas diferentes como autores, si bien todas ellas tienen en común y como punto central el énfasis en señalar que la reestructuración cognitiva está formada por un conjunto de estrategias terapéuticas que tratan de modificar los pensamientos de la persona, que serían responsables de ciertas conductas o emociones disfuncionales. El procedimiento estaría dirigido a que la persona, con la ayuda inicial del terapeuta, identifique, evalúe y discuta sus pensamientos desadaptativos, para sustituirlos por otros más apropiados de manera que se reduzca o elimine la perturbación emocional y/o conductual causada por los primeros (Bados, 2008). Por tanto, el objetivo principal de la técnica es identificar y modificar las cogniciones desadaptativas del cliente poniendo de relieve su impacto perjudicial sobre la conducta y las emociones. Por otra parte, se podría afirmar que la discusión o debate es el elemento o procedimiento terapéutico central de la técnica de reestructuración cognitiva. El término reestructuración cognitiva es confuso, ya que se ha utilizado indistintamente tanto para referirse a la técnica en su conjunto, como al debate propiamente dicho, como al proceso y al resultado del cambio "cognitivo". Sea cual fuese la acepción del término, la discusión o debate socrático es el elemento o procedimiento terapéutico central de la técnica de reestructuración cognitiva, a pesar de que los diversos enfoques incluyen otros muchos elementos. La relevancia del procedimiento de debate es tal que incluso se ha llegado a afirmar que es el núcleo de toda la terapia cognitiva, no solo de la reestructuración (Padesky, 1994; Padesky y Beck, 2003). La discusión o debate, salvando las diferencias entre las propuestas principales de la reestructuración cognitiva, trata en esencia de un diálogo, inicialmente del terapeuta con el cliente y, después, del cliente consigo mismo, que finaliza con la modificación de los pensamientos desadaptativos que provocaban en este emociones y conductas disfuncionales (Calero-Elvira et al, 2011; Dongil-Collado, 2008; Froján-Parga y Calero-Elvira, 2011). Sin embargo, de acuerdo con Clark y Egan (2015) se desconoce cómo operan estos procedimientos, pues han tenido poca investigación experimental y no se ha publicado una revisión sistemática de la literatura que haya concluido una definición empírica del diálogo socrático. Unido a esto, no se conocen con claridad los mecanismos de cambio del diálogo socrático o debate, existiendo gran discrepancia e incongruencia entre los teóricos acerca de la definición, el método, sus componentes y su uso (Carey y Mullan, 2004). Por tales razones, sería preferible ver la reestructuración cognitiva como un resultado más que un simple proceso.

A la vista de esto, es fácil concluir que no existen explicaciones consensuadas más allá de la de descripción del resultado; mucho menos hay explicaciones satisfactorias conductuales (de donde históricamente argumentan surgir) sobre los procesos básicos que ocurren con el cambio cognitivo cuando se utilizan los múltiples procedimientos de reestructuración cognitiva. 


\section{Defusión Cognitiva}

La defusión cognitiva es uno de los procedimientos más utilizados, especialmente dentro de la Terapia de Aceptación y Compromiso (ACT), una forma de terapia sobre la que se han realizado múltiples investigaciones y estudios de caso (Hayes, 2004). Es considerada por los mismos autores una evolución de la terapia cognitivo conductual tradicional y fuertemente enraizada en el conductismo radical.

La defusión cognitiva toma muchas formas, todas ellas dirigidas a no detener, controlar o modificar los eventos privados como los pensamientos, emociones, recuerdos, sensaciones, etc. (Barraca, 2011). La técnica clásica más conocida y utilizada es la de repetición (como leche-leche-leche), que básicamente consiste en repetir alguna palabra de manera constante durante largo tiempo hasta que pierde su sentido; se utilizan otros procedimientos con el mismo objetivo, tales como fisicalizar un pensamiento o algún evento privado, dándole forma o color para que la persona pueda verlo como algo ajeno y "en la distancia", (Hayes et al., 2006). Según estos autores, esa "toma de distancia" permite romper la identificación de la persona con el pensamiento (no es lo mismo decirse "soy malo" a "estoy teniendo el pensamiento de que soy malo"), alterando la literalidad de las relaciones verbales y su impacto en cuanto a su credibilidad; en palabras de los propios autores, se trataría de pasar del yo-aquí-ahora al yo-alli-entonces (Hayes, 2004; Hayes et al., 2006).

A diferencia de la reestructuración, no se dirige a cambiar el contenido de los pensamientos (su forma, frecuencia o duración), sino su función, socavando el dominio del contenido verbal sobre el comportamiento y detectando la fusión cognitiva (respuestas privadas equivalentes a hechos "físicos") que podría llevar a la evitación experiencial (comportamientos cuya función es el escape/evitación de dichas respuestas privadas desagradables o con funciones aversivas); pero al igual que la RC, en ocasiones se prefiere ver como un resultado por la variación de sus procedimientos específicos y sus distintos procederes. Uno de los argumentos que sostiene esta técnica es el relativo a la responsabilidad del contexto social-verbal en el impacto del significado literal del lenguaje con los objetos; es decir, que las palabras equivalen por "derivación arbitraria" a las propiedades inherentes de los mismos (fusión), paralelamente, se considera que es la imposición social-cultural la que enseña que algunos eventos privados son indeseables e insta a querer deshacerse de ellos (Hayes, 2004), de esta forma son las acciones y su propósito, es decir, las respuestas que llevan al escape/evitación de los pensamientos el verdadero problema, más no el contenido de los pensamientos. En este sentido, se señala la evitación de la experiencia como la causa de los problemas psicológicos cuando interviene para que una persona realice acciones, para la misma, importantes (López et al., 2010). En otras palabras, esta generación de terapias conductuales, en especial la ACT, afirma que las experiencias internas o privadas de una persona que causan malestar o que son desagradables no son sinónimo de enfermedad o psicopatología, sino que, al igual que el placer y el bienestar, son experiencias inherentes a la condición humana (Luciano y Valdivia, 2006); por tal motivo, no pretenden desaparecer ni cambiar la forma de los pensamientos. Contraría a la evitación experiencial, es la aceptación o el "dejar estar", sin embargo, los métodos que utilizan para lograr esto siguen siendo confusos a un nivel de análisis conceptual; sería, por ejemplo, el caso de la aceptación, que al ser un término de nivel medio que no se explica de forma conductual ha pasado como explicación del cambio y no algo a explicar, entonces pareciera ser un constructo mentalista cuando se establece como un paso intermedio necesario para la acción. Lo anterior también sucede con algunas otras aproximaciones terapéuticas que utilizan los términos medios como forma de explicación sin traducirse en un lenguaje conductual (Callaghan y Follette, 2020).

Las terapias contextuales como la ACT consideran que estos eventos privados no pueden ser modificados de la misma forma que lo que ocurre fuera de la piel (una asunción ontológica que parece ser contraria al monismo del conductismo radical, funcionalmente hablando). Por esta cuestión, ACT apuesta entonces por cambiar ese significado alterando la función de los mismos a través de los procedimientos de defusión. Es decir, cambia la forma en que las personas se relacionan con esos eventos privados creando contextos en los que las funciones que no son útiles se reducen. Como dijimos antes, ACT está directamente relacionada con la Teoría de los Marcos Relacionales, una teoría acerca del lenguaje y la cognición que defiende que las personas, por medio de la socialización, aprenden a relacionarse y a comparar eventos entre sí mediante relaciones arbitrarias y bidireccionales (Hayes et al, 2006). Las múltiples señales o claves contextuales 
controlan el tipo de marco relacional e indican las funciones psicológicas que se "derivan" de ello, y estas se caracterizan por tres propiedades básicamente: relaciones mutuas, relaciones combinatorias y la transformación de funciones. Esta última es la que consideran necesaria para comprender la psicopatología humana y sobre la que descansa la defusión cognitiva (Barnes-Holmes, 2004). Según algunos autores de esta teoría, la fusión o literalidad del pensamiento entre las palabras o eventos privados y su función surgiría como un resultado de comportarse verbalmente entre marcos bidireccionales y de transformación de funciones $(\mathrm{Gu}-$ tiérrez et al., 2005).

\section{Mindfulness}

Autores como Kabat - Zinn (2003) definen al mindfulness como "prestar atención de manera intencional al momento presente y sin juzgar", observando y dirigiendo la conciencia a todos los pensamientos, emociones y sensaciones que se presentan durante la práctica sin responder de manera reactiva y centrar la atención en aspectos del ambiente; la redirección de la atención no se hace únicamente a través de la meditación, si no con cualquier otra actividad que permita a una persona llegar a ese estado, dado que esta atención es contraria a la evitación de la experiencia (Kumar, 2002). El mindfulness, según sus teóricos, consiste en un entrenamiento para que las personas puedan llegar a ser capaces de admitir sus pensamientos y emociones, sin juzgarlos y sin intentar cambiarlos, bajo la premisa de que nadie es responsable de lo que piensa, pero sí de lo que hace. El procedimiento consiste en dirigir la atención hacia eventos privados o a determinadas actividades y ser consciente de lo que se está pensando o haciendo, dedicando unos minutos al día a "escuchar el cuerpo" sin importar la actividad que se esté realizando en ese momento (Moñivas et al., 2012; Parra et al., 2012).

Una intervención típica de mindfulness es la que se lleva a cabo en el programa de Reducción del Estrés Basado en Mindfulness -MBSR- por sus siglas en inglés; consiste en un estándar (que puede variar dependiendo de la población a la que se dirija) de 26 horas de sesión a lo largo de 8 semanas, más una sesión de 6 horas durante todo el día de un fin de semana con la finalidad de reducir el dolor, el estrés y el sufrimiento (Carmody y Baer, 2009). En cuanto a las terapias de tercera generación, las técnicas de mindfulness que se emplean son diversas: una de ellas es el body scan, que consiste en centrar y dirigir la atención al momento presente a través de la observación de la respiración y las sensaciones corporales mientras se acepta cualquier pensamiento que surja sin juzgarlo (Ussher et al., 2012). Otra forma y de las más sencillas es la llamada respirar y caminar conscientemente, que consiste en caminar y notar la respiración junto a los movimientos y sensaciones de los pies, el hacer atención plena al comer o cualquier otra actividad (Parra et al., 2012). La atención plena también puede considerarse un estado y no un rasgo, promovido por varias actividades como la misma meditación, donde algunos de sus efectos empíricamente respaldados han sido observados en la regulación emocional, disminución de la reactividad y mayor flexibilidad, beneficios interpersonales, empatía, compasión, habilidades de consejería y la reducción del estrés y ansiedad (Davis y Hayes, 2011).

Sin embargo, algunos críticos como Orgilés y Sevilla (2013), explican que a pesar de que el mindfulness cuenta con cierta evidencia a favor para el tratamiento de la reducción de la ansiedad, el dolor y del estrés, no existe esa misma evidencia en el resto de sus aplicaciones (Hofmann et al., 2010; Kumar, 2002); incluso, se considera que hay escasez de investigación y los metaanálisis realizados han arrojado resultados modestos, con debilidades metodológicas tales como grupos de muestra demasiados pequeños, ausencia de grupo control, falta de control de variables, dudas acerca de la validez de constructo, etc. Si bien algunos metaanálisis concluyen que el mindfulness ofrece aportaciones prometedoras, sugieren más ensayos clínicos, pruebas más rigurosas, comparación con otras intervenciones y la identificación de mecanismos conceptualmente sólidos (Baer, 2003; Bishop, 2002; Chiesa y Serretti, 2009; Grossman et al., 2004; Hofmann et al., 2010; Melbourne Academic Mindfulness Interest Group, 2006; Miró et al., 2011; Toneatto y Nguyen, 2007). Además, aunque la palabra mindfulness en español sea traducida como "atención plena" no hay evidencia suficiente que demuestre su efecto sobre la atención, por lo que la forma en la que actúa debería definirse operacionalmente (Bishop, 2002). 


\section{Propuesta explicativa basada en los principios de aprendizaje asociativo y seleccionista}

Las explicaciones anteriores, a nuestro ver, parten de una perspectiva dualista y, por tanto, incompatible con un planteamiento conductual. Desde una perspectiva analítico-conductual, esencialmente monista, sería necesario proponer una explicación alternativa basada en el aprendizaje respondiente y operante, y ello es posible si se entienden como procedimientos verbales olvidando la serie de inferencias necesarias (y arbitrarias) para hipotetizar la existencia de la cognición como algo distinto de la conducta verbal encubierta (Froján et al., 2017; Hall, 2001; Ruíz-Sancho et al., 2013; Tonneau, 2002). Es decir, se toma en cuenta que dichas técnicas operan sobre comportamientos privados pero verbales, que no difieren ontológicamente de los comportamientos públicos y que, por supuesto, están sujetas a las contingencias del proceso terapéutico, sin necesidad de recurrir a constructos hipotéticos.

\section{Propuesta alternativa para la Reestructuración Cognitiva}

Es incuestionable que la técnica de reestructuración cognitiva, y de manera específica el debate o dialogo socrático funcionan y tienen múltiples aplicaciones sobre distintos problemas clínicos. Sin embargo, no se conoce por qué funciona, ya que los supuestos constructos hipotéticos que se utilizan para explicar su éxito resultan indemostrables. Las críticas que se le han hecho a esta técnica en particular y a la modificación de conducta cognitiva en general se centran en la falta de clarificación de la relación entre cogniciones, respuestas emocionales y conducta motora (Salzinger, 1992) y la utilización de constructos mal operativizados (creencias irracionales, esquemas cognitivos), que conducen a modelos de causación circular.

Existen en la actualidad propuestas explicativas de la eficacia del método socrático que se basan en el estudio de los procesos de aprendizaje cuya ocurrencia es facilitada por tal procedimiento. La explicación conductual del método socrático como un proceso combinado de moldeamiento y encadenamiento junto con aspectos pavlovianos derivados del uso del lenguaje como sistema referencial ha sido y es, desde hace varios años, una plausible propuesta explicativa fundamentada en trabajos de investigación, que abarca todo el proceso terapéutico (Calero-Elvira et al., 2013; Follette et al., 1996; Froján, Vargas et al., 2010; Froján-Parga y Calero-Elvira, 2011; Froján et al., 2017).

La consideración del lenguaje y las verbalizaciones como el verdadero objetivo de la reestructuración permite el análisis de la interacción terapéutica que tiene lugar durante la aplicación de la técnica atendiendo a las funciones de dichas verbalizaciones, sin entenderlas como el producto de algo interno o profundo o la causa de otros comportamientos (Virués-Ortega y Froján-Parga, 2015).

Considerar de esta manera el proceso de reestructuración permite un análisis experimental de los procesos en los que se basa y, por consiguiente, aumentar la eficacia terapéutica. Las investigaciones actuales han concluido que el moldeamiento de la conducta verbal es una potente técnica de cambio de la conducta, no solo porque en las situaciones clínicas es más fácil moldear lo que se dice que lo que se hace, sino porque hay evidencia acerca de que la conducta verbal moldeada puede corresponderse mejor con el comportamiento a que se refiere que la conducta instruida o informada (Catania et al., 1990; Kohlenberg et al., 1993; Luciano, 1999).

Partiendo de estos resultados, se propone el estudio del dialogo socrático, como una forma de moldeamiento y encadenamiento verbal, donde aquellos cambios en las verbalizaciones son lo relevante a la hora de medir la eficacia de las técnicas. La cuestión propuesta es la siguiente: si tomamos en cuenta que la conducta privada (pensamientos, emociones) tiene el mismo estatus ontológico que el comportamiento público y se rige por los mismos principios, entonces la conducta privada verbal puede ser modificada con los mismos procedimientos de condicionamiento (Catania, 1974; Froján-Parga y Calero-Elvira, 2011; Luciano, 1999).

Durante el desarrollo del debate el terapeuta va cumpliendo distintas funciones a la vez que va reforzando diferencialmente (por sus efectos observados) las aproximaciones sucesivas hacia una forma de verbalización final deseada, cambiando o elevando el criterio de la verbalización a reforzar gradualmente (los llamados "pensamientos racionales"). En otras palabras, el procedimiento de moldeamiento verbal consiste en propiciar las condiciones para que se emita una verbalización, mientras que el terapeuta refuerza las verbalizaciones 
(clases de respuesta) que se vayan acercando cada vez más a la verbalización final. De igual forma, aquellas verbalizaciones que se alejan o son contrarias a la final deseada se van extinguiendo o castigando bajo una definición funcional.

Pero además otro proceso, en este caso respondiente, acompaña al moldeamiento típico del debate socrático. Autores como Mowrer (1954) ofrecen ejemplos de estos procesos de aprendizaje asociativo en el significado de las palabras relacionado con fenómenos de tipo social, viendo el estudio del lenguaje por derecho propio como una forma de "portar" y transferir significados, previamente aprendidos, de un signo a otro. Estos trabajos ofrecen una alternativa más parsimoniosa y sólida al estudio del lenguaje, su función y la transferencia de funciones en psicoterapia. Desde esta perspectiva, las palabras adquieren su significado a través de procedimientos de condicionamiento clásico, de manera que una palabra antes neutra puede adquirir la capacidad de elicitar una respuesta (privada), y esa misma palabra ahora condicionada, puede "transferir" la capacidad de elicitación a otra nueva con el mismo proceso y, de ahí, al estímulo físico al que se refiere (Froján et al., 2017). Para muchos autores, esta sería la explicación más plausible de la llamada función simbólica del lenguaje (Gutiérrez y Benjumea, 2003; Stemmer, 2001; Pereira et al., 2019; Tonneau; 2001).

Cuando se van moldeando esas verbalizaciones que son objetivo conductual o final/deseado, al mismo tiempo se van creando reglas verbales, es decir, guías que influyen en el comportamiento y que incluyen una contingencia de tres términos que ayudan al usuario a emitir los comportamientos deseados. A la vez, sabiendo que los comportamientos operantes y pavlovianos convergen en un mismo espacio y tiempo, el moldeamiento de verbalizaciones objetivo va emparejando o correlacionando de manera pavloviana la conducta verbal del cliente con expresiones y verbalizaciones contingentes del terapeuta, lo cual va transfiriendo otras funciones a dichas verbalizaciones tanto discriminativas como de reforzador positivo y negativo. Es decir, no solo se refuerza o castiga una respuesta verbal del cliente, sino que además las verbalizaciones del terapeuta, evocadas de manera contingente, condicionan las verbalizaciones sobre otros comportamientos del cliente de manera apetitiva o aversiva; esto puede ser aprovechado para que en momentos posteriores, emitiendo verbalizaciones que anticipen cierta consecuencia (históricamente condicionada) apetitiva funcione como operación motivadora aumentando la probabilidad del seguimiento de instrucciones fuera de la sesión (De Pascual y Trujillo, 2018; Froján, Alpañés et al., 2010).

\section{Propuesta alternativa para la Defusión Cognitiva y el Mindfulness}

Algunos autores han señalado que la defusión cognitiva (técnica dirigida a modificar el comportamiento simbólico) y la TMR sobre la que se asienta, pueden explicarse por medio de alternativas más parsimoniosas que las que proponen sus teóricos y que deben ser integradas para la comprensión de los fenómenos verbales y la equivalencia funcional (Tonneau, 2004; Tonneau et al., 2006).

Tonneau (2002) considera que la TMR carece de claridad teórica: es vaga y oscura y que incluso aquello que pretende explicar puede ser absorbido por el aprendizaje asociativo pavloviano de una manera más parsimoniosa. Este mismo autor ha sostenido debates con Hayes y Barnes-Holmes, argumentando que una lista (incluso larga) de procedimientos y de intuiciones no equivale a tener una teoría, y que, además si la TMR no puede explicar fenómenos básicos como lo hace el condicionamiento pavloviano, cómo esperar explicar fenómenos más complejos del comportamiento humano con solidez teórica y experimental. Otros autores como Malott (2003) y Palmer (2004) argumentan que la TMR no añade algo nuevo a lo anteriormente explicado en una contingencia de tres términos, incluso hacen una crítica a la definición ofrecida de operante generalizada, indicando que es una distinción vaga sobre si los marcos relacionales son una clase de comportamiento o si los marcos relacionales son parte de la historia que emite esa clase de comportamientos; además ponen en duda si la TMR se trata de un principio fundamental del comportamiento mostrando como puede emitirse una operante sin entrenamiento directo bajo explicaciones menos confusas conceptualmente.

No hay mucha diferencia en cuento a los procedimientos verbales descritos en líneas anteriores que funcionan en las técnicas utilizadas en las terapias contextuales, tales como la defusión y el mindfulness. En el caso de la defusión, a pesar de que históricamente se han realizado estudios y experimentos con resultados promete- 
dores, concretamente en los ejercicios de repetición de palabras en voz alta y de forma rápida (del tipo "leche, leche" que comentamos antes) que tienen un impacto emocional valorado como negativo en vez de suprimir la palabra, con la finalidad de alterar o desaparecer su significado literal (Masuda et al., 2010); también hay otros estudios que han resaltado las deficiencias metodológicas de los estudios originales (De Young et al., 2010; López de Uralde-Selva y Valero-Aguayo, 2021). En concreto, se señala que la atribución de ciertos procesos o mecanismos que explican la ocurrencia de la defusión no está correctamente fundamentada, sugiriendo que podrían operar distintos procesos en la disminución de la aversión y credibilidad de las palabras repetidas, tales como la pérdida de significado por extinción o la transferencia de funciones, explicaciones que, a juicio de sus autores, son más parsimoniosas. Además, se han realizado otros estudios donde el procedimiento de defusión en cuanto a repetición de palabras no brinda los resultados esperados y no hay diferencias significativas cuando se compara con otros procedimientos y un grupo control, aunado a que si los argumentos sobre la eficacia de dicha técnica datan sobre la disminución de la frecuencia de aparición de las palabras es algo contrario a sus objetivos propuestos (Barrera et al., 2015; López de Uralde-Selva y Valero-Aguayo, 2021; Pilecki y McKay, 2012).

Esto abre la puerta a posibles explicaciones alternativas con base en procesos de aprendizaje asociativo y seleccionista, tanto pavloviano como operante: la exposición a dichas palabras auto-referenciadas negativamente daría lugar a un proceso de extinción tanto respondiente como operante, donde la repetición alta y rápida de las palabras rompe el emparejamiento entre la palabra (símbolo/semántica) y la respuesta (significado) (previamente condicionada pavlovianamente).

Lo anterior sucede debido a que la palabra condicionada, ya sea en primer o segundo orden (Jara et al., 2007; Pereira et al., 2019; Tonneau, 2004), se presenta en múltiples ocasiones sin la respuesta incondicionada (aquello a lo que se refiere), al tiempo que se impide la evitación o el escape al forzar la exposición masiva al estímulo verbal; dicha exposición sin posibilidad de escape impide el reforzamiento, lo que conduce a la extinción. Con ello se abre la posibilidad de formar nuevos aprendizajes operantes y emparejamientos concordantes con los objetivos terapéuticos. Además, es posible que suceda el contracondicionamiento o trasferencia de funciones cuando se empareja con una nueva estimulación o respuesta apetitiva, recibiendo reforzamiento por mantener dicho contacto. Una vez más, observamos que, aunque se separan en el discurso con fines didácticos, en los procedimientos terapéuticos las respuestas operantes y respondientes están presentes de manera conjunta y entrelazada.

Por lo que respecta al mindfulness, hay problemas adicionales añadidos ya que, como hemos visto, su definición es imprecisa al igual que su utilidad. De hecho, resulta difícil operacionalizar la práctica del mindfulness cuando no se recurre a explicaciones funcionales y solo se describe su topografía, es decir, la técnica en sí y no para qué o cuál es su propósito. De igual forma, la mayoría de las investigaciones de esta estrategia terapéutica están basadas en la eficacia, más no en los mecanismos que la hacen funcionar (Moscoso, 2018), hipotetizándose distintos mecanismos explicativos tales como la exposición, relajación, mecanismos cognitivos, metacognición, percepción, aceptación, auto-observación, compasión, intención, actitud, regulación emocional, etc. (Hayes, 2002; Orgilés y Sevillá, 2013; Shapiro et al., 2006), propuestas todas ellas que, lejos de clarificar, empañan más la precisión teórica.

Por ejemplo, se suele comentar que el mindfulness no es una estrategia dirigida a la relajación per se, sin embargo, uno de los principales procedimientos del mindfulness es a través de ejercicios respiratorios lentos y focalizados. Es difícil no concluir que uno de los efectos del mindfulness se deba a un grado de relajación, siendo que a través de la respiración lenta y profunda y atendiendo a las sensaciones que se producen se pueden conseguir estados fisiológicos incompatibles con la actividad simpática del sistema nervioso, es decir, un incremento en la actividad de la división parasimpática que lleva a cambios fisiológicos del organismo propios del estado de relajación, tales como la reducción del ritmo respiratorio, cardiaco y la presión arterial (Moscoso, 2018; Nyklíček et al., 2013; Pace et al., 2009). Uno de los argumentos en contra de la relajación como mecanismo de mindfulness, es que en ocasiones este mismo lejos de relajar a una persona dispara aquellas sensaciones de malestar, estrés y ansiedad, pero este es un efecto ampliamente conocido en algunas formas de relajación también (Blanco et al., 2014; Furukawa y Sakano, 2007).

En ocasiones, las estrategias de mindfulness se han explicado también como una forma de defusión cognitiva, pues se trata de "dejar estar" a los pensamientos sin intentar cambiarlos (Barraca, 2011). Para estos casos, 
cuando esa es la intención del mindfulness, bien se podría aplicar la misma explicación alternativa sugerida de la defusión cognitiva que se ha comentado en párrafos anteriores, dando paso a la extinción de asociaciones pavlovianas previamente aprendidas y permitiendo el condicionamiento de otras nuevas asociaciones por medio de la transferencia de funciones.

Sea cual sea el objetivo o utilidad del mindfulness, debido a sus diversas aplicaciones, se debería indagar su función analíticamente para tener aproximaciones funcionales más certeras. Incluso los mecanismos de acción que se han hipotetizado dentro del mismo mindfulness son ambiguos, pues algunos son incompatibles con otros. En conclusión, parece ser que el mindfulness apuesta a una topografía y que puede utilizarse para distintos propósitos funcionales. De no proponerse explicaciones que vayan más allá de la descripción y especifiquen la función, caeríamos en una ficción explicativa característica de un argumento circular.

\section{Conclusión}

Si bien está fuera de toda duda que la Reestructuración Cognitiva (dialogo socrático), Defusión Cognitiva y Mindfulness, utilizadas ampliamente en las terapias cognitivo conductuales y las terapias contextuales, respectivamente, son (en su mayoría) altamente eficaces, consideramos que ocurre todo lo contrario con respecto a su claridad conceptual y capacidad explicativa. La inclusión de variables mediacionales a las explicaciones del comportamiento con el fin o intención de mejorar el conocimiento sobre la conducta humana pueden ser bien intencionadas, pero difícilmente aportan la claridad que se buscaba. Al integrar variables mediacionales para la explicación del comportamiento también se integraron problemas filosóficos, metodológicos, teóricos y prácticos, dado que dichas variables se consideraban de naturaleza distinta a la ya conocida del comportamiento (Burgos, 2004).

De esta forma, las explicaciones de las técnicas discutidas con variables mediacionales resultan en parte insatisfactorias por los problemas ya mencionados, además de la escasa evidencia de sus mecanismos llevando a la necesidad de formular explicaciones alternativas operantes y pavlovianas que coincidan con aquellas asunciones que han facilitado la manipulación de las variables que permiten mayor efectividad y replicación en la modificación del comportamiento clínicamente relevante. Si vemos estas técnicas como una forma de moldeamiento verbal y transferencia de funciones aportan una visión que facilita el rigor experimental, parsimonioso, aplicado, analítico, tecnológico y conceptualmente sistemático que se sugiere en el análisis de la conducta (Baer et al., 1987).

Otros podrían argumentar que las explicaciones mediacionales, al surgir históricamente después de los paradigmas operantes y respondientes significan progreso por su modernidad (después de todo son nuevas generaciones), y que regresar a las bases funcionales significa un retroceso; pero todo ello no es equivalente a solidez teórica o avance científico, lo cual se debe priorizar (Freixa y Froján, 2014; Valero, 2006).

Es entonces que las aproximaciones analítico-conductuales, el conductismo radical y los paradigmas respondientes y operantes suponen una ventaja a aquello que consideramos debilidades y problemáticas que se han encontrado en las explicaciones clásicas de las técnicas discutidas, ofreciendo explicaciones funcionales sin recurrir a constructos hipotéticos.

Insistimos en repetir que no se afirma que las terapias cognitivo-conductuales y las terapias contextuales, y con ello las técnicas que sugieren, sean acientíficas o que no sean útiles: todo lo contrario. Se insiste en la existencia de una amplia discrepancia entre las teorías mediacionales y las explicaciones de procesos básicos. El terapeuta que conozca los principios de aprendizaje que operan en su quehacer terapéutico, no solo añade una fuerte base teórica y una sólida fundamentación experimental (Froján-Parga y Calero-Elvira, 2011), sino que además podrá conocer y manipular variables como forma de aumentar la efectividad de su intervención. Teniendo como punto de partida conocer qué procesos ocurren en la interacción terapéutica y por qué funciona una técnica cuando es aplicada permite que los terapeutas sean más eficientes, más allá de los años de experiencia y los títulos (Froján et al., 2011). Aún con todo lo anterior, invitamos a los lectores a que lean y conozcan las propuestas y explicaciones tradicionales de las técnicas comentadas, pues seguramente encontraran cierta utilidad en ello. 
A manera de cierre, podemos utilizar la misma metáfora que emplean los contextualistas para dar cuenta del funcionamiento de sus propuestas terapéuticas y de cómo un clínico puede servirse de ellas (afirmando que es necesaria la TMR). La metáfora consiste en que la terapia es como conducir un automóvil, tú puedes conducir y llegar a tu destino, pero a veces, el automóvil se estropeará; si conoces el motor, podrás arreglarlo y seguir, si no, ahí te estancarás. Desde un estudio de procesos de la conducta verbal, agregaríamos que antes de saber arreglar el motor hay que conocer las piezas que lo integran y la posición en la que tienen que ir, sino cualquier intento de arreglo podría ser inútil.

\section{Referencias}

APA Presidential Task Force on Evidence-Based Practice (2006). Evidence-based practice in psychology. American Psychologist, 61(4), 271-285. https://doi.org/10.1037/0003-066X.61.4.271

Arbinaga-Ibarzábal, F. (2000). La revista análisis y modificación de conducta: 25 Años de historia en la psicología española. Análisis y Modificación de Conducta, 26(109), 619-646.

Bados, A. (2008). Terapia cognitiva de Beck. En F. J. Labrador (Coord.), Técnicas de modificación de conducta (pp. 517-533). Pirámide.

Baer, D. M., Wolf, M. M. y Risley, T. R. (1987). Some still-current dimensions of Applied Behavior Analysis. Journal of Applied Behavior Analysis, 20(4), 313-327. https://doi.org/10.1901/jaba.1987.20-313

Baer, R. A. (2003). Mindfulness training as a clinical intervention: A conceptual and empirical review. Clinical Psychology: Science and Practice, 10(2), 125-143. https://doi.org/10.1093/clipsy.bpg015

Barnes-Holmes, Y., Barnes-Holmes, D., McHugh, L. y Hayes, S. C. (2004). Relational frame theory: Some implications for understanding and treating human psychopathology. International Journal of Psychology and Psychological Research, 4(2), 355-375.

Barraca, J. (2011). ¿Aceptación o control mental? Terapias de aceptación y mindfulness frente a las técnicas cognitivo-conductuales para la eliminación de pensamientos intrusos. Análisis y Modificación de Conducta, 37(155-156), 43-63. https://doi.org/10.33776/amc.v37i155-156.1317

Barrera, T. L., Szafranski, D. D., Ratcliff, C. G., Garnaat, S. L. y Norton, P. J. (2015). An experimental comparison of techniques: Cognitive defusion, cognitive restructuring, and in-vivo exposure for social anxiety. Behavioural and Cognitive Psychotherapy, 44(2), 249-254. https://doi.org/10.1017/S1352465814000630

Bishop, S. R. (2002). What do we really know about Mindfulness-Based Stress Reduction? Psychosomatic Medicine, 64(1), 71-84. https://doi.org/10.1097/00006842-200201000-00010

Blanco, C., Estupiñá, F. J., Labrador, F. J., Fernández-Arias, I., Bernardo-de-Quirós, M. y Gómez, L. (2014). Uso de técnicas de relajación en una clínica de psicología. Anales de Psicología, 30(2), 403-411. https://doi.org/10.6018/analesps.30.2.158451

Burgos, J. E. (2004). Comentario: ¿Es la Teoría del Marco Relacional inteligible?. Acta Comportamentalia; Revista Latina de Análisis del Comportamiento, 12(1), 53-73.

Butler, A. C., Chapman, J. E., Forman, E. M. y Beck, A. T. (2006). The empirical status of cognitive behavioral therapy: A review of meta-analyses. Clinical Psychology Review, 26(1), 17-31. https://doi.org/10.1016/j.cpr.2005.07.003

Calero-Elvira, A., Froján-Parga, M. X., Ruíz-Sancho, E. M. y Vargas-de la Cruz, I. (2011). Conducta verbal de terapeutas y pacientes durante la aplicación de los distintos componentes de la reestructuración cognitiva. Revista Mexicana de Psicología, 28(2), 133-150. https://www.redalyc.org/articulo.oa?id=243029631003

Calero-Elvira, A., Froján-Parga, M. X., Ruíz-Sancho, M. E. y Alpañés-Freitag, M. (2013). Descriptive study of the socratic method: Evidence for verbal shaping. Behavior Therapy, 44(4), 625-638. https:// doi.org/10.1016/j.beth.2013.08.001

Callaghan, G. M. y Follette, W. C. (2020). Interpersonal Behavior Therapy (IBT), functional assessment, and the value of principle-driven behavioral case conceptualizations. The Psychological Record 70(4), 625-635. https://doi.org/10.1007/s40732-020-00395-1 
Carey, T. A. y Mullan, R. J. (2004). What is Socratic Questioning? Psychotherapy: Theory, Research, Practice, Training, 41(3), 217-226. https://doi.org/10.1037/0033-3204.41.3.217

Carmody, J. y Baer, R. A. (2009). How long does a Mindfulness-Based Stress Reduction program need to be? A review of class contact hours and effect sizes for psychological distress. Journal of Clinical Psychology, 65(6), 627-638. https://doi.org/10.1002/jclp.20555

Caro, I. (2013). Lo cognitivo en psicoterapias cognitivas una reflexión crítica. Boletín de Psicología, 107, 37-69. https://www.uv.es/seoane/boletin/previos/N107.htm

Catania, A. C. (1974). Investigación contemporánea en conducta operante. Trillas.

Catania, A. C., Matthews, B. A. y Shimoff, E. H. (1990). Properties of rule-governed behavior and their implications. En D. E. Blackman y H. Lejeune (Dirs.), Behavior analysis in theory and practice (pp. 215-230). LEA.

Chiesa, A. y Serretti, A. (2009). Mindfulness-Based Stress Reduction for stress management in healthy people: A review and meta-analysis. The Journal of the Alternative and Complementary Medicine, 15(5), 593-600. https://doi.org/10.1089/acm.2008.0495

Clark, G. y Egan, S. (2015). The socratic method in cognitive behavioral therapy: A narrative review. Cognitive Therapy and Research, 39(6), 863-879. https://doi.org/10.1007/s10608-015-9707-3

Davis, D. M. y Hayes, S. C. (2011). What are the benefits of mindfulness? A practice Review of Psychotherapy-related Research. Psychotherapy, 48(2), 198-208. https://doi.org/10.1037/a0022062

De Pascual, R. y Trujillo, C. (2018). Estudio de la relación entre las verbalizaciones motivadoras y el seguimiento de instrucciones en la terapia psicológica. Revista Clínica Contemporánea, 9(2), Artículo e14. https://doi.org/10.5093/cc2018a11

De Silva, P. (1984). Buddhism and behaviour modification. Behavior Research Therapy, 22(6), 661-678. https://doi.org/10.1016/0005-7967(84)90129-3

De Young, K. P., Lavender, J.M., Washington, L.A., Looby, A. y Anderson, D. A. (2010). A controlled comparison of the word repeating technique with a word association task. Journal of Behavior Therapy and Experimental Psychiatry, 41(4), 426-432. https://doi.org/10.1016/j.jbtep.2010.04.006

Dongil-Collado, E. (2008). Reestructuración cognitiva: Un caso de estrés postraumático. Ansiedad y Estrés, 14(2-3), 265-288.

Dymond, S., May, R. J., Munelly, A. y Hoon, A. E. (2010). Evaluating the evidence base for Relational Frame Theory: A citation analysis. The Behavior Analyst, 33(1), 97-117. https://doi.org/10.1007/BF03392206

Echeburúa, E., Salaberría, K., de Corral, P. y Polo-López R. (2010). Terapias psicológicas basadas en la evidencia: Limitaciones y retos del futuro. Revista Argentina de Clínica Psicológica, 19(3), 247-256.

Fatih, K. y Hakan, M. (2012). Aaron Temkin Beck (born july 18, 1921-) Biography. Journal of Cognitive-Behavioral Psychotherapy and Research, 1(2), 77-80.

Follette, W. C., Naugle, A. E. y Callaghan, G. M. (1996). A radical behavioural understanding of the therapeutic relationship in effecting change. Behavior Therapy, 27(4), 623-641. https://doi.org/10.1016/S00057894(96)80047-5

Freixa, E. (2003). ¿Qué es conducta? Revista Internacional de Psicología Clínica y de la Salud, 3(3), 595-613.

Freixa, E. y Froján, M. X. (2014). La falacia del argumento cronológico: Reflexiones acerca de la confusión entre modernidad y progreso y sus repercusiones sobre el desarrollo de la psicología. Conductual, Revista Internacional de Interconductismo y Análisis de la Conducta, 2(1), 13-22. http://www.conductual.com/articulos/La\%20falacia\%20del\%20argumento\%20cronologico.pdf

Froján-Parga, M. X. y Calero-Elvira, A. (2011). Guía para el uso de la reestructuración cognitiva como un procedimiento de moldeamiento. Behavioral Psychology/ Psicología Conductual, 19(3), 659-682.

Froján, M. X., Núñez de Prado, M. y De Pascual, R. (2017). Cognitive techniques and language: A return to behavioral origins. Psicothema, 29(3), 352-357.

Froján, M. X., Alpañés, M., Calero, E. y Vargas, I. (2010). Una concepción conductual de la motivación en el proceso terapéutico. Psicothema, 22(4), 556-561.

Froján, M. X., Vargas, I., Calero, A. y Ruíz, E. (2010). Categorización de la conducta verbal del cliente durante la reestructuración cognitiva. Análisis y Modificación de Conducta, 36(153-154), 105-114. https://doi. org/10.33776/amc.v36i153-154.1063 
Froján, M. X., Ruíz, E. M., Montaño, M., Calero, A. y Alpañés, M. (2011). Estudio comparativo de la conducta verbal del terapeuta según su experiencia durante la evaluación clínica. Anales de Psicología, 27(2), 311-318.

Furukawa, H. y Sakano, Y. (2007). Influence of personality traits and cognitions on autogenic training induced anxiety. Japanese Journal of Autogenic Therapy, 27(2), 1-10.

Gross, A. C. y Fox, E. J. (2009). Relational Frame Theory: An overview of the controversy. The Analysis of Verbal Behavior, 25(1), 87-98. https://doi.org/10.1007/BF03393073

Grossman, P., Niemann, L., Schmidt, S. y Walach, H. (2004). Mindfulness-Based Stress Reduction and health benefits a meta-analysis. Journal of Psychosomatic Research, 57(1), 35-43. https://doi.org/10.1016/S00223999(03)00573-7

Gutiérrez, M. T. y Benjumea, S. (2003). Formación de clases Funcionales utilizando un entrenamiento de condicionamiento clásico. Revista Latinoamericana de Psicología, 35(2), 165-174. http://hdl.handle. net/11441/16982

Gutierrez, O., Luciano, C. y Rodríguez, M. (2005). Análisis de los contextos verbales en el trastorno de evitación experiencial y en la Terapia de Aceptación y Compromiso. Revista Latinoamericana de Psicología, $37(2), 333-358$.

Hall, G. (2001). Mechanisms of functional equivalence. European Journal of Behavior Analysis, 2(1), 69-71. https://doi.org/10.1080/15021149.2001.11434176

Hayes, S. C. (2002). Buddhism and Acceptance and Commitment Therapy. Cognitive and Behavioral Practice, 9(1), 58-66. https://doi.org/10.1016/S1077-7229(02)80041-4

Hayes, S. C. (2004). Acceptance and Commitment Therapy, Relational Frame Theory, and the third wave of behavioral and cognitive therapies. Behavior Therapy, 35(4), 639-665. https://doi.org/10.1016/S00057894(04)80013-3

Hayes, S. C. (2006). Acceptance, mindfulness, and science. Clinical Psychology: Science and Practice, 9(1), 101-106. https://doi.org/10.1093/clipsy.9.1.101

Hayes, S. C., Luoma, J. B., Bond, F. W., Masuda, A. y Lillis, J. (2006). Acceptance and Commitment Therapy: Model, processes and outcomes. Behaviour Research and Therapy, 44(1), 1-25. https://doi.org/10.1016/j. brat.2005.06.006

Hernández-Pineda, J. A., Medina-Guerrero, E. A. y Castillo-Martínez, J. A. (2020). Cognitive-Behavioral Therapy: A theoretical review of its bases. Journal of Basic and Applied Psychology Research, 1(2), 20-24. https://doi.org/10.29057/jbapr.v1i2.5362

Hofmann, S. G., Sawyer, A. T., Witt, A. A. y Oh, D. (2010). The effect of mindfulness-based therapy on anxiety and depression: A meta-analytic review. Journal of Consulting and Clinical Psychology, 78(2), 169-183. https://doi.org/10.1037/a0018555

Jara, E., Maldonado, A. y Vila, J. (2007). Procesos de inferencia y aprendizaje de relaciones causales en el condicionamiento de segundo orden. Psicológica, 28(22), 215-236. https://www.uv.es/psicologica/articulos2.07/7JARA.pdf

Kabat-Zinn, J. (2003). Mindfulness-based interventions in context: Past, present and future. Clinical Psychology: Science and Practice, 10(2). 144-156. https://doi.org/10.1093/clipsy.bpg016

Kohlenberg, R. J., Tsai, M. y Dougher, M. J. (1993). The dimensions of clinical behavior analysis. The Behavior Analyst, 16(2), 271-282. https://doi.org/10.1007/BF03392636

Kumar, S. (2002). An introduction to buddhism for the cognitive-behavioral therapist. Cognitive and Behavioral Practice, 9(1), 40-43. https://doi.org/10.1016/S1077-7229(02)80038-4

Landriscini, N. (2006). La Terapia Racional Emotiva de Albert Ellis. Norte de Salud Mental, 6(25), 126-129.

Larrubia, A. (2019). MBI (Mindfulness Based Intervention). Desarrollo profesional y salud laboral. RIECS, Revista de Investigación y Educación en Ciencias de la Salud, 4(1), 2530-2787. https://doi.org/10.37536/ RIECS.2019.4.S1.122

Llobell, J., Frías, M. D. y Monterde, H. (2004). Tratamientos psicológicos con apoyo empírico y práctica clínica basada en la evidencia. Papeles del Psicólogo, 25(87), 1-8.

López de Uralde-Selva, M. A. y Valero-Aguayo, L. (2021). Cognitive defusion as a verbal exercise: An experimental approach. Psicothema,33(1), 77-85. https://doi.org/10.7334/psicothema2020.103 
López, J. C., Ruiz, F. J., Feder, J., Barbero, A., Suárez J. J., Rodríguez, J. A. y Luciano, C. (2010). The role of experiential avoidance in the performance on a high cognitive demand task. International Journal of Psychology and Psychological Therapy, 10(3), 475-488. https://www.ijpsy.com/volumen10/num3/275/the-role-ofexperiential-avoidance-in-the-EN.pdf

Luciano, M. C. (1999). Terapia de aceptación y compromiso (ACT) y Psicoterapia analítica funcional (FAP). Fundamentos, características y precauciones. Análisis y modificación de conducta, 25(102), 497-584.

Luciano, M. C. y Valdivia, M. S. (2006). La Terapia de Aceptación y Compromiso (ACT). Fundamentos, características y evidencia. Papeles del Psicólogo, 27(2), 79-91.

Luciano, M. C., Gutiérrez, O. y Rodríguez, M. (2005). Análisis de los contextos verbales en el trastorno de evitación experiencial y en la terapia de aceptación y compromiso. Revista Latinoamericana de Psicología, 37(2), 333-358.

Malott, R. C. (2003). Behavior analysis and linguistic productivity. The Analysis of Verbal Behavior, 19(1), 11-18. https://doi.org/10.1007/BF03392978

Mañas, I. (2007). Nuevas terapias psicológicas: La tercera ola de terapias de conducta o terapias de tercera generación. Gaceta de Psicología, 40, 26-34. https://hdl.handle.net/20.500.12008/21755

Masuda, A., Twohig, M. P., Stormo, A. R., Feinstein, A. B., Chou, Y. -Y. y Wendell, J. W. (2010). The effects of cognitive defusion and thought distraction on emotional discomfort and believability of negative self-referential thoughts. Journal of Behavior Therapy and Experimental Psychiatry, 41(1), 11-17. https://doi. org/10.1016/j.jbtep.2009.08.006

Melbourne Academic Mindfulness Interest Group (2006). Mindfulness-based psychotherapies: A review of conceptual foundations, empirical evidence and practical considerations. Australian and New Zealand Journal of Psychiatry, 40(4), 285-294. https://doi.org/10.1080/j.1440-1614.2006.01794.x

Miró, M. T., Perestelo-Pérez, L., Pérez, J., Rivero, A., González, M. de la Fuente, J. y Serrano, P. (2011). Eficacia de los tratamientos basados en mindfulness para los trastornos de ansiedad y depresión: Una revisión sistemática. Revista de Psicopatología y Psicología Clínica, 16(1), 1-14. https://doi.org/10.5944/rppc. vol.16.num.1.2011.10347

Moñivas, A., García-Diex, G. y García-de-Silva, R. (2012). Mindfulness (atención plena): Concepto y teoría. Portularia Revista de Trabajo Social, 12(extra), 83-89.

Moore, J. (1995). Radical behaviourism and the subjective-objective distinction. The Behavior Analyst, 18(1), 33-49. https://doi.org/10.1007/BF03392690

Moscoso, M. (2018). Mindfulness, autorregulación emocional y reducción de estrés: ¿Ciencia o ficción? Revista Mexicana de Investigación en Psicología, 10(2), 101-121.

Mowrer, O. H. (1954). The psychologist looks at language. American Psychologist, 9(11), 660-694. https:// doi.org/10.1037/h0062737

Nyklíček, I., Mommersteeg, P. M., Van Beugen, S., Ramakers, C. y Van Boxtel, G. J. (2013). Mindfulness-Based Stress Reduction and physiological activity during acute stress: A randomized controlled trial. Health Psychology, 32(10), 1110-1113. https://doi.org/10.1037/a0032200

Oller, J. (2009). El contraguión de Albert Ellis: Su influencia en la terapia racional emotiva conductual. Revista de Análisis Transaccional y Psicología Humanista, 61(2), 238-254.

Orgilés, M. L. y Sevilla, J. (2013). ¿Por qué no soy un terapeuta mindfulness?. Informació Psicológica, 106(1), 54-69. http://dx.medra.org/10.14635/IPSIC.2014.106.5

Pace, T. W., Negi, L. T., Adame, D. D., Cole, S. P., Sivilli, T. I., Brown, T. D., Issa, M. J. y Raison, C. L. (2009). Effect of compassion meditation on neuroendocrine, innate immune and behavioral responses to psychosocial stress. Psychoneuroendocrinology, 34(1), 87-98. https://doi.org/10.1016/j.psyneuen.2008.08.011

Padesky, C. A. (1994). Schema change processes in cognitive therapy. Clinical Psychology and Psychotherapy, 1(5), 267-278. https://doi.org/10.1002/cpp.5640010502

Padesky, C. A. y Beck, A. T. (2003). Science and Philosophy: Comparison of Cognitive Therapy and Rational Emotive Behavior Therapy. Journal of Cognitive Psychotherapy: An International Quarterly, 17(3), 211-224. https://doi.org/ 10.1891/jcop.17.3.211.52536 
Palmer, D. C. (2004). Generic response classes and Relational Frame Theory: Response to Hayes and Barnes-Holmes. Journal of the Experimental Analysis Behavior, 82(2). 225-234. https://doi.org/10.1901/ jeab.2004.82-225

Palmi, J. y Solé, S. (2016). Intervenciones basadas en mindfulness (atención plena) en psicología del deporte. Revista de Psicología del Deporte, 25(1), 147-155. http://hdl handle.net/10459.1/56778

Parra, M., Montañés, J., Montañés, M. y Bartolomé, R. (2012). Conociendo mindfulness. ENSAYOS, Revista de la Facultad de Educación de Albacete, 27, 29-46. https://doi.org/10.18239/ensayos.v27i0.130

Pereira, G. L., Ricote, A., de Pascual-Verdú, R. y Froxán-Parga, M. X. (2019). Los procesos de condicionamiento clásico en la interacción verbal terapéutica. Revista Mexicana de Análisis de la Conducta, 45(1), 90-110. https://doi.org/10.5514/rmac.v45.i1.70870

Pérez-Álvarez, M. (2001). Afinidades entre las nuevas terapias de conducta y las terapias tradicionales con otras orientaciones. Revista Internacional de Psicología Clínica y de la Salud, 1(1), 15-33.

Pérez-Álvarez, M. (2006). La terapia de conducta de tercera generación. eduPsykhé, 5(2), 159-172. https:// journals.ucjc.edu/EDU/article/view/3781

Pilecki, B. y McKay, D. (2017). An experimental investigation of cognitive defusion. The Psychological Record, 62(1), 19-40. https://doi.org/10.1007/BF03395784

Ruíz-Sancho, E., Froján-Parga, M. X. y Calero-Elvira, A. (2013). Análisis de la conducta verbal del cliente durante el proceso terapéutico. Anales de Psicología, 29(3), 779-790. https://doi.org/10.6018/analesps.29.3.135401

Ryle, G. (2005). El concepto de lo mental (E. Rabossi, trad.). Ediciones Paidós. (Original publicado en 1949). Salzinger, K. (1992). Cognitive therapy: a misunderstanding of B. F. Skinner. Journal of Behavior Therapy and Experimental Psychiatry, 23(1), 3-8. https://doi.org/10.1016/0005-7916(92)90019-F

Shapiro, S. L., Carlson, L. E., Astin, J. A. y Freedman, B. (2006). Mechanisms of mindfulness. Journal of Clinical Psychology, 62(3), 373-386. https://doi.org/10.1002/jclp.20237

Skinner, B. F. (1985). Cognitive science and behaviourism. British Journal Psychology, 76(3), 291-301. https://doi.org/10.1111/j.2044-8295.1985.tb01953.x

Solé, S., Carrança, B., Serpa, D. y Palmi J. (2014). Aplicaciones del mindfulness (conciencia plena) en lesión deportiva. Revista de Psicología del Deporte, 23(2), 01-508. http://hdl handle.net/10459.1/60373

Stemmer, N. (2001). Pavlovian correlations and action frames. European Journal of Behavior Analysis, 2(1), 94-98. https://doi.org/10.1080/15021149.2001.11434184

Stevens, K. y Méndez, D. (2007). Nuevas herramientas para la psicología clínica: Las terapias basadas en la evidencia. Cuadernos de Neuropsicología/ Panamerican Journal of Neuropsychology, 1(3), 265-272. http:// pepsic.bvsalud.org/scielo.php?pid=\$0718-41232007000300009\&script=sci abstract\&tlng=en

Toneatto, T. y Nguyen, L. (2007). Does mindfulness meditation improve anxiety and mood symptoms? A review of the controlled research. Le Revue Canadienne de Psychiatrie, 52(4), 260-266. https://doi. org/10.1177/070674370705200409

Tonneau, F. (2001). Equivalence relations: A critical analysis. European Journal of Behavior Analysis, 2(1), 1-33. https://doi.org/10.1080/15021149.2001.11434165

Tonneau, F. (2002). Who can understand Relational Frame Theory? A reply to Barnes-Holmes and Hayes. European Journal of Behavior Analysis, 3(2), 95-102. https://doi.org/10.1080/15021149.2002.11434209

Tonneau, F. (2004). Verbal understanding and pavlovian processes. The Behavior Analyst Today, 5(2), 158169. https://doi.org/10.1037/h0100029

Tonneau, F., Arreola, F. y Martínez, A. G. (2006). Function transformation without reinforcement. Journal of the Experimental Analysis of Behavior, 85(3), 393-405. https://doi.org/10.1901/jeab.2006.49-05

Tortosa, F. y Mayor, L. (1997). Evolución de las técnicas de reducción de ansiedad: Del conductismo al cognitivismo. Ansiedad y Estrés, 3(2-3), 135-154.

Ussher, M., Spatz, A., Copland, C., Nicolaou, A., Cargill, A, Amini-Tabrizi, N. y McCracken, L. M. (2012). Immediate effects of a brief mindfulness-based body scan on patients with chronic pain. Journal of Behavioral Medicine, 37(1), 127-134. https://doi.org/10.1007/s10865-012-9466-5 
Valadez, A. (2002). Aplicación de técnicas cognitivo conductuales en un caso de problemas familiares: Reestructuración cognitiva, asertividad, y manejo de contingencias. Revista Electrónica de Psicología Iztacala, 5(2), Artículo e3. http://www.revistas.unam.mx/index.php/repi/article/view/22668/21407

Valero, L. (2016). La extensión del laboratorio de la práctica clínica: generalización formal o funcional. Acta Comportamentalia: Revista Latina de Análisis del Comportamiento, 24(2), 135-153.

Vera-Villarroel, P. (2004). Estrategias de intervención en psicología clínica: Las intervenciones apoyadas en la evidencia. Liberabit Revista Peruana de Psicología, 10, 4-10. http://ojs3.revistaliberabit.com/publicaciones/revistas/RLE 101 estrategias-de-intervencion-en-psicologia-clinica-las-intervenciones-apoyadas-en-la-evidencia.pdf

Virués-Ortega, J. y Froján-Parga, M. X. (2015). A translational approach to the functional analysis of language in psychotherapy. International Journal of Clinical and Healt Psychology, 15(1), 69-75. https://doi. org/10.1016/j.ijchp.2014.10.001

Zilio, D. y Carrara, K. (2008). Mentalismo e explicação do comportamento: aspectos da crítica behaviorista radical à ciência cognitiva. Acta Comportamentalia: Revista Latina de Análisis del Comportamiento, 16(3), 399-417.

Artículo recibido: 04/11/2020

Artículo aceptado: 26/10/2021 